\title{
Lithium-rich giants in the Galactic thick disk ${ }^{\star}$
}

\author{
L. Monaco ${ }^{1}$, S. Villanova ${ }^{2}$, C. Moni Bidin ${ }^{2}$, G. Carraro ${ }^{1}$, D. Geisler ${ }^{2}$, P. Bonifacio $^{3}$, O. A. Gonzalez ${ }^{4}$, \\ M. Zoccali ${ }^{5}$, and L. Jilkova ${ }^{1,6}$
}

\author{
${ }^{1}$ European Southern Observatory, Casilla 19001, Santiago, Chile \\ e-mail: Imonaco@eso.org \\ 2 Universidad de Concepción, Casilla 160-C, Concepción, Chile \\ 3 GEPI, Observatoire de Paris, CNRS, Université Paris Diderot, Place Jules Janssen, 92190 Meudon, France \\ 4 ESO, Karl-Schwarzschild-Strasse 2, 85748 Garching, Germany \\ 5 Departamento Astronomía y Astrofísica, Pontificia Universidad Católica de Chile, Av. Vicuña Mackenna 4860 Stgo., Chile \\ ${ }^{6}$ Department of Theoretical Physics and Astrophysics, Faculty of Science, Masaryk University, Kotlářská 2, 61137 Brno, \\ Czech Republic
}

Received 8 December 2010 / Accepted 24 February 2011

\begin{abstract}
Context. Lithium is a fragile element, which is easily destroyed in the stellar interior. The existence of lithium-rich giants still represents a challenge for stellar evolution models.

Aims. We have collected a large database of high-resolution stellar spectra of 824 candidate thick-disk giants having 2 MASS photometry and proper motions measured by the Southern Proper-Motion Program (SPM). In order to investigate the nature of Li-rich giants, we searched this database for giants presenting a strong Li I resonance line.

Methods. We performed a chemical abundance analysis on the selected stars with the MOOG code along with proper ATLAS-9 model atmospheres. The iron content and atmospheric parameters were fixed by using the equivalent width of a sample of Fe lines. We also derive abundances for $\mathrm{C}, \mathrm{N}$, and $\mathrm{O}$ and measure or derive lower limits on the ${ }^{12} \mathrm{C} /{ }^{13} \mathrm{C}$ isotopic ratios, which is a sensible diagnostic of the stars evolutionary status.

Results. We detected five stars with a lithium abundance higher than 1.5, i.e. Li-rich according to the current definition. One of them (SPM-313132) has $A(\mathrm{Li})>3.3$ and, because of this, belongs to the group of the rare super Li-rich giants. Its kinematics makes it a likely thin-disk member and its atmospheric parameters are compatible with it being a $4 M_{\odot}$ star either on the red giant branch (RGB) or the early asymptotic giant branch. This object is the first super Li-rich giant detected at this phase. The other four are likely low-mass thick-disk stars evolved past the RGB luminosity bump, as determined from their metallicities and atmospheric parameters. The most evolved of them lies close to the RGB-tip. It has $A(\mathrm{Li})>2.7$ and a low ${ }^{12} \mathrm{C} /{ }^{13} \mathrm{C}$ isotopic ratio, close to the cool bottom processing predictions.
\end{abstract}

Key words. stars: abundances - stars: atmospheres - stars: chemically peculiar - Galaxy: disk

\section{Introduction}

Lithium is a fragile element, which is easily destroyed in the stellar interiors at temperatures higher than $\sim 2.5 \times 10^{6} \mathrm{~K}$. As soon as a star evolves to the red giant phase, the convective envelope deepens and bring to the stellar surface material that has been exposed to high temperatures in the stellar interior, causing an overall dilution of $\mathrm{Li}$ in a giant's atmosphere. Red giant stars are, therefore, expected to present a low amount of Li. Starting from the meteoritic abundance of $A(\mathrm{Li})^{1}=3.3 \mathrm{dex}($ Grevesse \& Sauval 1998), standard models predict the dilution of $\mathrm{Li}$ in the giants' atmosphere down to a level of about 1.5 dex, depending on the stellar mass and metallicity (Iben 1967a,b). Giants having $\mathrm{A}(\mathrm{Li})$ exceeding this value are usually termed $\mathrm{Li}$-rich and represent a challenge for standard stellar evolution models.

About $1-2 \%$ of K-giants are Li-rich, according to the above definition (Brown et al. 1989; Smith et al. 1995). In order to explain their existence, it was suggested that dilution might not have been effective in these stars or that the atmospheric $\mathrm{Li}$

^ Based on observations taken at the Las Campanas and La Silla/ Paranal observatory (ESO proposal ID: 077.B-0348).

${ }^{1} A(\mathrm{Li})=\log \frac{n(\mathrm{Li})}{n(\mathrm{H})}+12$. abundance might have been increased by the ingestion of a planet or a brown dwarf because of the expansion of the stellar atmosphere (Siess \& Livio 1999). Alternatively, a Li production phase following the first dredge-up dilution has been proposed.

The possibility that Li-rich giants avoided dilution and simply preserved their initial lithium abundance is contradicted, however, by the low ${ }^{12} \mathrm{C} /{ }^{13} \mathrm{C}$ carbon isotopic ratios measured (da Silva et al. 1995), which suggests that mixing has taken place in those stars. Moreover the existence of a few stars with $\mathrm{Li}$ abundances exceeding the meteoritic value ("super" Li-rich giants, e.g. Balachandran et al. 2000) argue against a preservation of the primordial $\mathrm{Li}$ abundance. At the same time, the ingestion of a planet or a brown dwarf would also lead to an increase of the ${ }^{9} \mathrm{Be}$ abundance, which is not observed in Li-rich giants (Melo et al. 2005).

On the other hand, there is agreement among the investigators that $\mathrm{Li}$ can be enhanced in the giant's atmosphere through the Cameron \& Fowler (1971, hereafter CF71) ${ }^{7}$ Be-transport mechanism. In order for this mechanism to be effective, ${ }^{3} \mathrm{He}$ should first be circulated from the stellar atmosphere down to regions at temperatures high enough for ${ }^{3} \mathrm{He}$ burning through the ${ }^{3} \mathrm{He}(\alpha, \gamma){ }^{7} \mathrm{Be}$ reaction. Then, the produced ${ }^{7} \mathrm{Be}$ should be circulated up to the stellar surface where it can decay into ${ }^{7} \mathrm{Li}$ 
by electron capture: ${ }^{7} \mathrm{Be}\left(\mathrm{e}^{-}, v\right)^{7} \mathrm{Li}$. In luminous $\left(M_{\mathrm{bol}}=-6\right.$ to -7 ) intermediate mass asymptotic giant branch (AGB) stars, the H-burning shell is in contact with the stellar convective envelope and, therefore, ${ }^{3} \mathrm{He}$ burning takes place partially under convective conditions (hot-bottom burning). In low-mass stars, the convective envelope and the H-burning shell are, however, not in touch and the existence of a mixing mechanism has to be postulated to connect material from those two zones.

Besides the standard mixing caused by the first dredge-up, an additional extra-mixing episode is known to take place at the luminosity bump along the red giant branch (RGB) in lowmass stars (Gratton et al. 2004). Charbonnel \& Balachandran (2000, hereafter CB00) took advantage of Hipparcos parallaxes to accurately place Li-rich giants in the Hertzspung-Russell diagram, and revealed that some stars were in the process of completing the dilution and were, hence, erroneously classified as Li-rich. Besides those stars, truly Li-rich giants tend to cluster into two groups: at the RGB-bump luminosity in low-mass stars and at the early AGB in intermediate mass stars. At these phases the mean molecular weight discontinuity left over by the first dredge-up is erased, allowing the occurrence of extra-mixing processes. Most of the Li-rich giants present, however, ${ }^{12} \mathrm{C} /{ }^{13} \mathrm{C}$ compatible with first dredge-up predictions (CB00). The Li-rich stage would then be produced by a relatively shallow extramixing episode, a precursor of the extra-mixing phenomenon that further lowers the ${ }^{12} \mathrm{C} /{ }^{13} \mathrm{C}$ isotopic ratio in more evolved stars (see Charbonnel \& Do Nascimento 1998, CB00). After the stellar atmosphere is enriched in lithium, convection begins to again dilute it by exposing surface material to the higher temperatures of the stellar interiors. Therefore, the Li-rich phase would be by nature a short-lasting phase, which agrees with the low number of detected stars. ${ }^{12} \mathrm{C} /{ }^{13} \mathrm{C}$ isotopic ratios lower than first dredge-up prediction may indicate that the dilution process has started again and Li should be lower than the peak value.

Charbonnel \& Balachandran (2000) certainly provide a valuable framework for the investigation of Li-rich giants. Nevertheless, the authors do not embrace the whole complex phenomenology of Li-rich giants. For instance, HD 77361 is a super Li-rich star lying close to the RGB-bump, but with ${ }^{12} \mathrm{C} /{ }^{13} \mathrm{C}=4$ (Kumar \& Reddy 2009), i.e. significantly lower than expected from first dredge-up models. Li-rich low-mass giants were also detected along the upper RGB/AGB in globular clusters (Kraft et al. 1999; Smith et al. 1999), the Galactic Bulge (Uttenthaler et al. 2007; Gonzalez et al. 2009) and dwarf spheroidal galaxies (dSph, Domínguez et al. 2004; Monaco \& Bonifacio 2008). In particular, Domínguez et al. (2004) and Uttenthaler et al. (2007) studied a sample of C stars in the Draco $\mathrm{dSph}$ and in the Galactic Bulge, respectively. In these cases, the high Li abundance detected in a few stars was attributed to enrichment during third dredge-up episodes in thermally pulsating AGB stars. Notice also that Li-rich stars detected in globular clusters and in the the tidal stream of the Sagittarius dSph (Monaco \& Bonifacio 2008) have low mass and lie either close to the tip of the RGB or on the AGB. As such, they do not belong to the second category of Li-rich giants identified by CB00.

Rotation has often been claimed to be the culprit for extramixing processes that happen along the RGB (Sweigart \& Mengel 1979). Fekel \& Balachandran (1993) found that many rapidly rotating $\left(v \sin i=6-46 \mathrm{~km} \mathrm{~s}^{-1}\right)$, chromospherically active giants present high Li abundances. They argued that the high observed rotational velocities are the results of momentum transferred to the surface from the stellar interior. This transfer may be accompanied by the dredge-up of ${ }^{7} \mathrm{Be}$, whose decay increases the surface Li abundance. Indeed, while K-giants are usually slow rotators, Drake et al. (2002) concluded that the fraction of Li-rich giants may rise from the standard 1-2\% up to $\sim 50 \%$ when rapid rotators are considered $\left(v \sin i>8 \mathrm{~km} \mathrm{~s}^{-1}\right)$.

Using IRAS colors, de La Reza et al. (1996, 1997) also noted that many Li-rich stars presented far-infrared excess and speculated that the Li-rich phase was associated with a massloss episode. A few Li-rich giants certainly present evidence of mass loss and chromospheric activity (Balachandran et al. 2000; Drake et al. 2002). Fekel \& Watson (1998) and Jasniewicz et al. (1999), however, analyzed samples of giants with farinfrared color excess and did not detect any additional Li-rich star. Therefore, mass loss may or may not be associated with the Li-rich phase.

Several models were proposed to interpret the Li-rich giant phenomenon. While the classical Cameron-Fowler mechanism was envisaged to take place in AGB stars (CF71) during He-shell flashes, the "cool bottom processing" takes place in RGB stars and can go on for an extended period of time as the star climbs up the RGB (Sackmann \& Boothroyd 1999). In order to be able to produce ${ }^{7} \mathrm{Li}$, it requires deep mixing with a high-speed $\dot{M}_{\mathrm{p}}$, typically $\dot{M}_{\mathrm{p}} \gtrsim 10^{-7} M_{\odot} \mathrm{yr}^{-1}$, but often as fast as $10^{-4} M_{\odot} \mathrm{yr}^{-1}$. According to the computations of Sackmann \& Boothroyd (1999), the ${ }^{7} \mathrm{Li}$ in the envelope increases as time on the RGB passes, reaches a plateau, and then decreases again, as the freshly formed $\mathrm{Li}$ is mixed back into high-temperature regions where it is destroyed. Thus the Li-rich phase is limited in time. The level of Li-enrichment depends on the mixing speed as well as on the mixing geometry, i.e. the relative surfaces of upward and downward streams.

According to Denissenkov \& Weiss (2000) and Denissenkov \& Herwig (2004), enhanced extra-mixing may be triggered by the episodic ingestion of a massive planet or a brown dwarf (see Carlberg et al. 2010). This could happen at any time along the RGB. The ingested body would naturally contribute its momentum to the stellar structure and, after the enhanced extra-mixing gave rise to the Li-rich phenomenon, lithium would start to be destroyed again by convection. This model would explain the existence of Li-rich giants all along the RGB at luminosities different from the RGB-bump and the connection with stars with high rotational velocities, but would neither explain the clustering of giants at the RGB-bump in low-mass stars nor the existence of Li-rich giants with low rotational velocities.

Other possible sources for the extra-mixing episodes required to produce Li-rich stars were identified in thermohaline mixing (Charbonnel \& Zahn 2007) and magneto-thermohaline mixing (Denissenkov et al. 2009). Guandalini et al. (2009, hereafter GPBU09) investigated the latter case and showed that both Li-rich and Li-poor RGB/AGB stars can be accounted for by models of extra-mixing induced by magnetic buoyancy. Their investigation does not try to interpret super Li-rich giants $(A(\mathrm{Li})>$ $2.5 \mathrm{dex}$ ) and still depends on several ad-hoc assumptions, yet it provides a self-consistent interpretation for the apparent clustering of Li-rich stars at the RGB-bump, as well as the presence of Li-poor stars and the few Li-rich stars in the late RGB. The clump of Li-rich stars detected on the early AGB by CB00 were interpreted by GPBU09 as stars that actually are on the RGB. The first detection of a variable magnetic field in the Li-rich giant HD 232862 has been recently made (Lèbre et al. 2009).

Given the complexity of the problem, it is mandatory to collect more data to better clarify the observational framework. Unfortunately, the intrinsic rarity of Li-rich giants requires a dedicated search for these kind of objects, which demands extensive observational campaigns. 
Table 1. Log of the observations for the program stars.

\begin{tabular}{lccccccc}
\hline \hline SPM & 2MASS & Date & $\begin{array}{c}\text { Exposure time } \\
(\mathrm{s})\end{array}$ & $\lambda / \Delta \lambda$ & Instrument & Telescope & Observatory \\
\hline 142173 & $00321256-3834022$ & $2006-09-13$ & 1800 & 38000 & Echelle & 2.5 m Irénée du Pont & Las Campanas \\
171877 & $00392022-3131354$ & $2006-08-23$ & 900 & 48000 & FEROS & 2.2 m ESO-MPI & La Silla \\
225245 & $00544638-2735304$ & $2006-09-11$ & 2400 & 38000 & Echelle & $2.5 \mathrm{~m}$ Irénée du Pont & Las Campanas \\
$313132^{a}$ & $01202066-3409541$ & $2006-09-12$ & 300 & 38000 & Echelle & $2.5 \mathrm{~m}$ Irénée du Pont & Las Campanas \\
$343555^{b}$ & $01294200-3015464$ & $2006-09-12$ & 850 & 38000 & Echelle & $2.5 \mathrm{~m}$ Irénée du Pont & Las Campanas \\
\hline $\mathrm{HD}_{787^{c}}$ & $00120998-1756177$ & $2006-08-20$ & 100 & 48000 & FEROS & $2.2 \mathrm{~m}$ ESO-MPI & La Silla \\
\hline
\end{tabular}

Notes. The SPM and 2MASS identification numbers are also reported together with the spectral resolution of the obtained observations. ${ }^{(a)}$ Alternative ID: CD-34 510. ${ }^{(b)}$ High-velocity star. ${ }^{(c)}$ Alternative ID: HR37.

Table 2. Coordinates, photometry, reddening and proper motions (pm) of the program stars.

\begin{tabular}{lcccccccccc}
\hline \hline SPM & $\alpha(\mathrm{J} 2000)$ & $\delta(\mathrm{J} 2000)$ & $\begin{array}{c}l \\
\mathrm{deg}\end{array}$ & $\begin{array}{c}b \\
\mathrm{deg}\end{array}$ & $K$ & $J-K$ & $E(B-V)$ & $E(J-K)$ & $\begin{array}{c}p m(\mathrm{RA}) \\
\mathrm{mas} / \mathrm{yr}\end{array}$ & $\begin{array}{c}p m(\mathrm{Dec}) \\
\mathrm{mas} / \mathrm{yr}\end{array}$ \\
\hline 142173 & $00: 32: 12.56$ & $-38: 34: 02.3$ & 321.099 & -77.874 & 9.29 & 0.78 & 0.018 & 0.009 & $-1.2 \pm 2.0$ & $-19.4 \pm 1.6$ \\
171877 & $00: 39: 20.23$ & $-31: 31: 35.5$ & 333.144 & -84.872 & 8.12 & 0.91 & 0.019 & 0.010 & $6.8 \pm 1.6$ & $-7.7 \pm 2.5$ \\
225245 & $00: 54: 46.38$ & $-27: 35: 30.4$ & 245.156 & -89.126 & 8.79 & 0.88 & 0.014 & 0.007 & $4.5 \pm 1.2$ & $-3.3 \pm 1.1$ \\
313132 & $01: 20: 20.66$ & $-34: 09: 54.1$ & 263.253 & -80.616 & 7.04 & 0.73 & 0.031 & 0.016 & $26.4 \pm 3.1$ & $-5.2 \pm 2.4$ \\
343555 & $01: 29: 42.01$ & $-30: 15: 46.4$ & 235.664 & -81.047 & 8.46 & 0.72 & 0.017 & 0.009 & $27.9 \pm 1.7$ & $-25.3 \pm 1.3$ \\
\hline HD 787 & $00: 12: 09.99$ & $-17: 56: 17.8$ & 76.321 & -77.101 & 1.85 & 0.85 & 0.029 & 0.015 & & \\
\hline
\end{tabular}

In the context of a survey of the Galactic thick disk, we constructed a large database of high-resolution, high-quality echelle spectra of giant stars (Carraro et al. 2005; Moni Bidin 2009; Moni Bidin et al. 2010). By screening this database we serendipitously detected the five Li-rich giants we present in the present work. The paper is organized as follows: in Sect. 2 we outline the target selection criterion and present the observations and data reduction performed with reference to the detected Li-rich stars. In Sect. 3 we present the performed abundance analysis on the selected stars and in Sect. 4 we present the measured target rotational velocities. In Sect. 5 we use the derived chemical abundances and atmospheric parameters together with their radial velocities and proper motions to investigate whether the kinematics of our stars are compatible with membership to the thick-disk. Finally, in Sects. 6 and 7 we discuss and summarize our findings.

\section{Observations and data reduction}

Our investigation is based on the sample defined by Girard et al. (2006, hereafter G06). It consists of 1196 red giant stars within 15 degrees from the south Galactic Pole, with infrared 2 MASS photometry (Skrutskie et al. 2006) and absolute proper motions from the SPM3 catalog (Girard et al. 2004).

The sample was defined from an IR color-magnitude diagram in the color range $0.7 \leq J-K \leq 1.1$, to select intermediate metallicity stars, i.e. thick-disk members. Contamination by halo stars should account for $\sim 8 \%$ of the selected objects (G06). The sample is confined to a cone perpendicular to the Galactic disk, which G06 estimated to be volume-complete up to at least $3 \mathrm{kpc}$ from the Galactic plane. A sloped-cutoff limit at faint magnitudes avoided contamination by dwarf stars, except for the closest ones $(d \leq 63 \mathrm{pc})$.

High-resolution, high-quality echelle spectra were acquired with four different instruments for a total of 824 stars belonging to the G06 sample during 38 observing nights between 2005 and 2007. Telescopes with different collecting powers were employed to efficiently secure spectra with roughly the same resolution and quality for targets spanning a wide range of magnitudes $(5 \leq V \leq 16)$.
We inspected by eye all spectra in the database in the region around the lithium resonance doublet at $6707.8 \AA$. The line was detected in several stars, but only the five objects in Table 2 presented a strong line. Indeed, the Li subordinate line at $6103.6 \AA$ was detected in these stars only. A detailed analysis of all objects with detected $\mathrm{Li}$ lines will be presented elsewhere. We focus here on the five stars that alone present strong lithium lines.

Stars discussed here were observed either with the Echelle/ duPont or the FEROS/2.2 m spectrographs. Below we briefly summarize the data reduction performed with reference to these observations. Details can be found in Moni Bidin (2009) and Moni Bidin et al. (2010). In Table 1 we present the SPM identification number (which will be used henceforth), 2 MASS designations, date of the observations, instruments, observatory, exposure times, and resolutions for our stars. Table 2 reports the target coordinates, the 2 MASS infrared magnitudes, the SPM3 proper motions and the reddening as derived from the Schlegel et al. (1998) maps.

During each run we observed several bright standard stars with accurate parameters (radial and rotational velocities, fundamental parameters and metallicity) available in the literature. Among them, the well known Li-rich giant HD 787 (Castilho et al. 2000) was also observed. This star is analyzed here as well.

Data reduction was performed using standard $\mathrm{IRAF}^{2}$ tasks and included bias, flat-fielding, order tracing, background and sky subtraction, and extraction. Standard Th-Ar arcs were used for the wavelength calibrations. For the duPont spectra we used "milky" flats, obtained with a diffusion filter set before the CCD. These frames provided the needed wavelengthindependent pixel-to-pixel response variation but, at odds with standard spectroscopic flat-field frames, the "milky" flats did not correct the fringing pattern that affected our spectra longward of about $7500 \AA$. For these, we created a fringing image from the Dome Flat, reduced as a normal spectrum and then normalized,

2 IRAF is distributed by the National Optical Astronomy Observatories, which are operated by the Association of Universities for Research in Astronomy, Inc., under cooperative agreement with the National Science Foundation. 
Table 3. Program stars atmospheric parameters.

\begin{tabular}{lccccccc}
\hline \hline SPM & $\begin{array}{c}T_{\text {eff }} \\
\mathrm{K}\end{array}$ & $\log g$ & $\begin{array}{c}\xi \\
\mathrm{km} \mathrm{s}^{-1}\end{array}$ & $A(\mathrm{Fe})$ & {$[\mathrm{Fe} / \mathrm{H}]$} & $\begin{array}{c}S / N \\
@ 670 \mathrm{~nm}\end{array}$ & $\begin{array}{c}v_{\text {helio }} \\
\mathrm{km} \mathrm{s}^{-1}\end{array}$ \\
\hline Sun & 5777 & 4.44 & 0.80 & 7.50 & & & \\
\hline 142173 & 4330 & 1.50 & 1.35 & 6.81 & -0.69 & 118 & $38.0 \pm 0.7$ \\
171877 & 3930 & 1.10 & 1.40 & 6.73 & -0.77 & 109 & $-15.8 \pm 0.5$ \\
225245 & 3920 & 0.65 & 1.50 & 6.51 & -0.99 & 110 & $3.9 \pm 0.6$ \\
313132 & 4530 & 2.00 & 1.20 & 7.51 & +0.01 & 42 & $7.7 \pm 0.6$ \\
343555 & 4530 & 2.25 & 1.00 & 6.88 & -0.62 & 106 & $119.8 \pm 0.6$ \\
\hline HD 787 & 4000 & 1.50 & 1.50 & 7.50 & +0.00 & $>150$ & $-6.0 \pm 0.9$ \\
HD 787 & 3870 & 0.85 & 1.27 & 7.54 & +0.04 & $>150$ & $-6.0 \pm 0.9$ \\
\hline
\end{tabular}

Notes. The spectra signal-to-noise ratios are also indicated, as well as the measured radial velocities. ${ }^{(a)}$ Atmospheric parameters are assumed from Castilho et al. (2000).

and we divided all science spectra by this image in the reddest orders, where the fringing pattern became visible. The resulting correction was deemed satisfactory.

The radial velocities (RVs) of the observed stars were measured with a cross-correlation (CC) technique (Tonry \& Davis 1979) by means of the fxcor IRAF task. Radial velocity standards acquired with the same instrument were used as templates for the CC. After the radial velocity measurements, all spectra were shifted to the laboratory wavelength. The IRAF task rvcorrect was used to calculate the Earth motion and convert the observed RVs to the heliocentric system. Measured RVs are reported in Table 3 as well as the spectral signal-to-noise $(S / N)$.

\section{Chemical abundance analysis}

For each target we obtained an initial estimate of the stellar effective temperature $\left(T_{\text {eff }}\right)$ from the dereddened infrared $(J-K)$ color along with the Alonso et al. (1999) calibrations. The reddening was derived from the COBE reddening maps (Schlegel et al. $1998)$, obtaining $E(J-K)$ from $E(B-V)$ by means of the transformations of Cardelli et al. (1989). The surface gravity $(\log g)$ was derived with the aid of theoretical isochrones (Marigo et al. 2008), adopting an age of 10 Gyr (see also Feltzing \& Bensby 2009), a metallicity $Z=0.004$, and a distance estimated from a photometric parallax, by assuming that target stars followed the same $(J-K)$ vs. K mean ridge line as the globular cluster 47 Tuc. Indeed, the thick disk age and metallicity are similar to those of this cluster (Wyse \& Gilmore 2005). The microturbulent velocity $\xi$ was evaluated from the derived gravity adopting the scaling relation: $\xi\left(\mathrm{km} \mathrm{s}^{-1}\right)=-0.254 \times \log g+1.930$ (Marino et al. 2008, hereafter M08).

These initial parameters were adopted to compute a proper model atmosphere for each star using the the Linux port of the ATLAS-9 code (Kurucz 1993; Sbordone et al. 2004). Equivalent widths (EWs) of a selected sample of iron lines (see M08) were measured with a Gaussian fitting. For each star, the measured EWs and the model atmosphere were then used within the MOOG $^{3}$ (Sneden 1973) local thermodynamic equilibrium (LTE) stellar line analysis code to refine the atmospheric parameters. In particular, the stellar $T_{\text {eff }}$ was fixed by imposing that the abundance derived for each line should be independent of the transition excitation potential. $\xi$ was estimated by minimizing the dependence of the abundances from the measured EWs, and $\log g$ by requiring that $\mathrm{Fe}$ I and $\mathrm{Fe}$ II lines gave the same iron abundance, within the errors. The stellar atmospheric parameters

\footnotetext{
$\overline{{ }^{3} \text { http://verdi.as.utexas.edu/moog.html }}$
}

eventually adopted are reported in Table 3 together with the derived iron abundance.

The abundance of $\mathrm{Li}, \mathrm{C}, \mathrm{N}, \mathrm{O}$, and the ${ }^{12} \mathrm{C} /{ }^{13} \mathrm{C}$ isotopic ratio were obtained with spectral synthesis of selected regions using the MOOG code along with an ATLAS-9 model atmosphere computed for each star with the final adopted atmospheric parameters. The atomic and molecular linelists were taken from the Kurucz database ${ }^{4}$.

For the hottest stars in the sample, i.e. \#142173, \#313132, and \#343555, it was possible to measure the carbon abundance from the $\mathrm{C}_{2}$ band at $5635.5 \AA$. For stars \#171877, \#225245 and HD 787 carbon was measured from the CH G-band at $4310 \AA$. For \#343555 we managed to measure carbon from both bands and obtained an abundance difference of 0.05 dex, which proves the consistency of the two measurements. Oxygen was measured by synthesis of the line at $6300 \AA$. The Ni I line at $6300.34 \AA$ was taken into account in the synthesis of the Oxygen line. For the Ni I line we adopted the $\log g f$ value derived by Johansson et al. (2003), namely $\log g f=-2.11$. The nitrogen abundance was derived by synthesis of the 8001-8005 $\AA$ spectral region, which is rich in $\mathrm{CN}$ lines. The same region was also used to set the ${ }^{12} \mathrm{C} /{ }^{13} \mathrm{C}$ isotopic ratio, thanks to presence of the ${ }^{13} \mathrm{CN}$ line at $\sim 8004.7 \AA$ and the ${ }^{12} \mathrm{CN}$ lines at 8003-8004 $\AA$. Owing to the low residual intensity of the ${ }^{13} \mathrm{CN}$ line, for stars \#142173, $\# 171877$, and \#343555 we provide only lower limits to the carbon isotopic ratio.

Because the C-N-O elements are partly bound together in molecules for stars cooler than $\sim 4500 \mathrm{~K}$, their abundances were derived together in an iterative process. Solar abundances for Fe, $\mathrm{C}, \mathrm{N}$, and $\mathrm{O}$ were obtained in the same way using the Kurucz solar spectrum for the purpose (Kurucz et al. 1984). The $\mathrm{C}_{2}$ band at $5635.5 \AA$ is not visible in the solar spectrum, so we used the $\mathrm{CH}$ G-band. The derived solar abundances are reported in Table 4.

Afterward, we measured the lithium abundances from both the resonance doublet at $6707.8 \AA$ and the subordinate line at $6103.6 \AA$. For the former, we adopted the Reddy et al. (2002) hyperfine structure. We also added to the linelist the two Fe I and Ti I lines at $6707.433 \AA$ and $6708.125 \AA$, respectively, listed in Gonzalez et al. (2009, hereafter G09). These lines are not present in the Kurucz database, but are available at the Vienna Atomic Line Database (VALD ${ }^{5}$, Kupka et al. 2000) and are required to obtain a good fit of the Li I doublet at $6707.8 \AA$. For these lines we adopted the oscillator strength ( $\log g f)$ calibrated on Arcturus by G09.

\footnotetext{
${ }^{4}$ http://kurucz.harvard.edu/

5 http://vald.astro.univie.ac.at/ vald/php/vald.php
} 
Table 4. Measured abundances for the program stars.

\begin{tabular}{lccccccccccc}
\hline \hline SPM & {$[\mathrm{Fe} / \mathrm{H}]$} & {$[\mathrm{C} / \mathrm{Fe}]$} & {$[\mathrm{N} / \mathrm{Fe}]$} & {$[\mathrm{O} / \mathrm{Fe}]$} & $\mathrm{C} / \mathrm{O}$ & ${ }^{12} \mathrm{C} /{ }^{13} \mathrm{C}$ & $\begin{array}{c}A(\mathrm{Li}) \\
670.8 \mathrm{~nm}\end{array}$ & $\begin{array}{c}A(\mathrm{Li}) \\
610.3 \mathrm{~nm}\end{array}$ & $\begin{array}{c}A(\mathrm{Li})_{\mathrm{NLTE}}{ }^{a} \\
670.8 \mathrm{~nm}\end{array}$ & $\begin{array}{c}A(\mathrm{Li})_{\mathrm{NLTE}}{ }^{a} \\
610.3 \mathrm{~nm}\end{array}$ & $\begin{array}{c}v \sin i \\
\mathrm{~km} \mathrm{~s}^{-1}\end{array}$ \\
\hline Sun & 7.50 & 8.49 & 7.95 & 8.83 & & & & & & & 2.72 \\
\hline 142173 & -0.69 & +0.02 & +0.02 & +0.20 & +0.30 & $>15$ & 2.80 & 2.52 & 2.73 & $2.2 \pm 0.7$ \\
171877 & -0.77 & -0.23 & +0.42 & +0.34 & +0.12 & $>15$ & 2.49 & 2.29 & 2.46 & 2.52 & $<1.8$ \\
225245 & -0.99 & -0.44 & +0.54 & +0.08 & +0.14 & 8 & 2.90 & 2.71 & 2.83 & 2.93 & $<1.1$ \\
313132 & +0.01 & +0.07 & +0.01 & -0.07 & +0.63 & 13 & 3.45 & 3.35 & 3.37 & 3.56 & $3.3 \pm 0.5$ \\
343555 & -0.62 & +0.09 & -0.08 & +0.19 & +0.36 & $>10$ & 1.79 & $<1.94$ & 1.94 & $<2.11$ & $2.5 \pm 0.8$ \\
\hline HD 787 & +0.00 & -0.24 & +0.33 & +0.02 & 0.21 & 15 & 2.03 & 2.14 & 2.09 & 2.40 & 2.40 \\
HD 787 & +0.04 & -0.38 & +0.09 & -0.22 & 0.27 & 15 & 1.77 & 2.12 & 1.98 & 2.40 \\
\hline
\end{tabular}

Notes. The measured stellar rotational velocities are also indicated. ${ }^{(a)}$ NLTE corrections are calculated according to Lind et al. (2009). Owing to the grid boundaries, we assume: $\xi=2.0 \mathrm{~km} \mathrm{~s}^{-1}$ for all stars; $T_{\text {eff }}=4000 \mathrm{~K}$ for \#171877, \#225245, and HD 787 (2nd instance); log $g=1.0$ for \#225245 and HD 787 (2nd instance); [Fe/H] = 0.0 for \#313132 and HD 787 (2nd instance). ${ }^{(b)}$ Atmospheric parameters are assumed from Castilho et al. (2000); C, N and O abundances are taken from Meléndez et al. (2008). Carbon isotopic ratio is taken from da Silva et al. (1995).

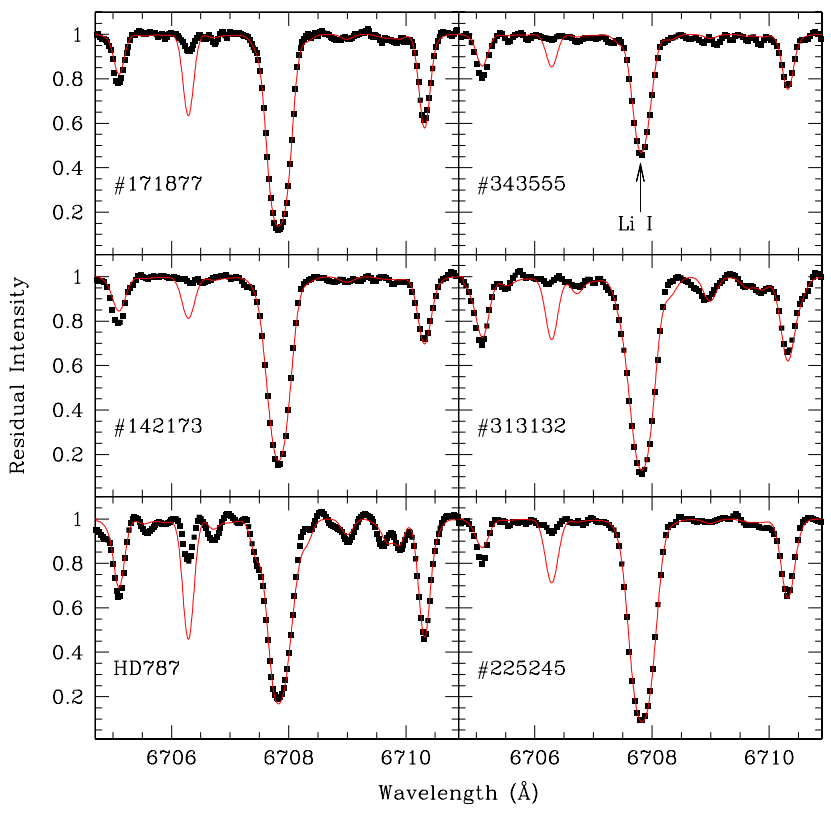

Fig. 1. Sample of the target stars' spectra in the region of the Li I resonance doublet.

Figures 1-3 present the spectra of our stars and HD 787 around the two LiI lines and in the 8001-8005 $\AA$ region. For each star, the best-fitting synthetic spectrum (continuous line) is superimposed on the observed one (filled squares). The measured $\mathrm{C}, \mathrm{N}, \mathrm{O}$, and $\mathrm{Li}$ abundances are reported in Table 4. For star \#343555, the lithium abundance was measured from the $6707.8 \AA$ line, while we derive an upper limit only from the subordinate line, which is barely detected in the observed spectrum.

The first of the two HD 787 entries in Table 4 reports the $\mathrm{Li}$ abundances we obtained adopting the atmospheric parameters in Castilho et al. (2000, see first HD 787 entry in Table 3). We derived Li abundances of 2.03 and 2.14 dex for the $6707.8 \AA$ and $6103.6 \AA$ lines, respectively. This agrees well with Castilho et al. (2000), who measured 2.0 and 2.2 dex (LTE analysis). These authors did not measure the $\mathrm{C}, \mathrm{N}$, and $\mathrm{O}$ abundances and, therefore, we adopted the Meléndez et al. (2008) values, which are also reported in the Table. We also report the ${ }^{12} \mathrm{C} /{ }^{13} \mathrm{C}$ isotopic ratio measured by da Silva et al. (1995).

For HD 787, however, we derive a slightly cooler temperature (see second HD787 entry in Table 3) with respect to Castilho et al. (2000). We therefore performed an independent

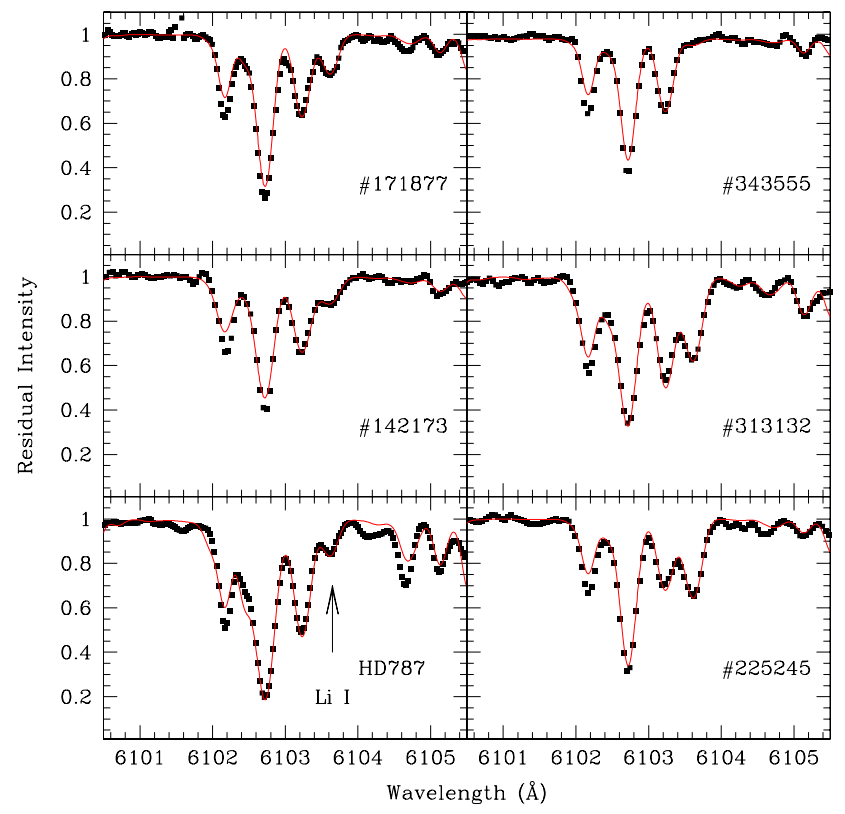

Fig. 2. Sample of the target stars' spectra in the region of the Li I subordinate line.

abundance analysis, following the same procedure as outlined above. The second HD 787 entry in Table 4 reports the C, N, $\mathrm{O}$, and $\mathrm{Li}$ abundances we derived for HD 787 with our analysis. The ${ }^{12} \mathrm{C} /{ }^{13} \mathrm{C}$ isotopic ratio we measured also perfectly agrees with da Silva et al. (1995).

Lind et al. (2009) calculated the correction to the Li abundances measured from both the resonance and the subordinate lines at $6707.8 \AA$ and $6103.6 \AA$ due to non-LTE effects (NLTE). We used their grid to evaluate the correction to apply to our measures. In Cols. 10 and 11 of Table 4 we report the Li abundances corrected for NLTE. These values have to be regarded only as referential. Indeed, owing to the grid boundaries, we had to adopt in the computation $\xi=2.0 \mathrm{~km} \mathrm{~s}^{-1}$ for all stars; $T_{\text {eff }}=4000 \mathrm{~K}$ for $\# 171877, \# 225245$, and HD 787 (2nd instance); $\log g=1.0$ for $\# 225245$ and HD 787 (2nd instance); $[\mathrm{Fe} / \mathrm{H}]=0.0$ for \#313132 and HD 787 (2nd instance).

We finally estimated the errors on absolute abundances and abundance ratios owing to the noise of the spectra and to the uncertainties on atmospheric parameters. For the ${ }^{12} \mathrm{C} /{ }^{13} \mathrm{C}$ ratio only the error owing to the spectral $S / N$ is significant because a change in temperature, gravity, or microturbulence does not alter 
Table 5. Estimated errors on absolute abundances and abundance ratios for the hottest (\#313132) and coolest (\#225245) stars in our sample.

\begin{tabular}{lccccc}
\hline \hline$\Delta(\mathrm{El})$ & $S / N$ & $\Delta T_{\text {eff }}=100$ & $\Delta \log (g)=0.20$ & $\Delta \xi=0.10 \mathrm{~km} \mathrm{~s}^{-1}$ & $\sigma_{\text {tot }}$ \\
\hline$\Delta[\mathrm{Fe} / \mathrm{H}]_{\# 313132}$ & 0.02 & +0.04 & +0.02 & -0.05 & 0.07 \\
$\Delta[\mathrm{Fe} / \mathrm{H}]_{\# 225245}$ & 0.01 & +0.01 & +0.03 & -0.05 & 0.06 \\
\hline$\Delta[\mathrm{C} / \mathrm{H}]_{\# 313132}$ & 0.03 & -0.02 & +0.04 & +0.00 & 0.05 \\
$\Delta[\mathrm{C} / \mathrm{H}]_{\# 225245}$ & 0.05 & -0.03 & +0.04 & +0.01 & 0.07 \\
\hline$\Delta[\mathrm{N} / \mathrm{H}]_{\# 313132}$ & 0.05 & -0.01 & +0.12 & -0.01 & 0.13 \\
$\Delta[\mathrm{N} / \mathrm{H}]_{\# 225245}$ & 0.08 & -0.10 & +0.13 & +0.00 & 0.18 \\
\hline$\Delta[\mathrm{O} / \mathrm{H}]_{\# 313132}$ & 0.05 & +0.01 & +0.09 & +0.00 & 0.10 \\
$\Delta[\mathrm{O} / \mathrm{H}]_{\# 225245}$ & 0.02 & +0.02 & +0.06 & +0.00 & 0.07 \\
\hline$\Delta\left({ }^{12} \mathrm{C} /{ }^{13}\right)_{\# 313132}$ & 5 & 0 & 0 & 0 & 5 \\
$\Delta\left({ }^{12} \mathrm{C} /{ }^{13}\right)_{\# 225245}$ & 2 & 0 & 0 & 0 & 2 \\
\hline$\Delta\left(\log \epsilon \mathrm{Li}_{670}\right)_{\# 313132}$ & 0.10 & +0.13 & +0.03 & -0.02 & 0.17 \\
$\left.\Delta(\log \epsilon \operatorname{Li})_{670}\right)_{\# 225245}$ & 0.08 & +0.15 & +0.03 & -0.05 & 0.18 \\
\hline$\Delta\left(\log \epsilon \mathrm{Li}{ }_{610}\right)_{\# 313132}$ & 0.03 & +0.10 & +0.00 & +0.00 & 0.10 \\
$\Delta\left(\log \epsilon \mathrm{Li}{ }_{610}\right)_{\# 225245}$ & 0.02 & +0.13 & -0.01 & +0.00 & 0.13 \\
\hline$\Delta[\mathrm{C} / \mathrm{Fe}]_{\# 313132}$ & 0.03 & -0.06 & +0.02 & +0.05 & 0.09 \\
$\Delta[\mathrm{C} / \mathrm{Fe}]_{\# 225245}$ & 0.05 & -0.04 & +0.01 & +0.06 & 0.09 \\
\hline$\Delta[\mathrm{N} / \mathrm{Fe}]_{\# 313132}$ & 0.05 & -0.05 & +0.10 & +0.04 & 0.13 \\
$\Delta[\mathrm{N} / \mathrm{Fe}]_{\# 225245}$ & 0.08 & -0.11 & +0.10 & +0.05 & 0.18 \\
\hline$\Delta[\mathrm{O} / \mathrm{Fe}]_{\# 313132}$ & 0.05 & -0.03 & +0.07 & +0.05 & 0.10 \\
$\Delta[\mathrm{O} / \mathrm{Fe}]_{\# 225245}$ & 0.02 & +0.01 & +0.03 & +0.05 & 0.06 \\
\hline
\end{tabular}

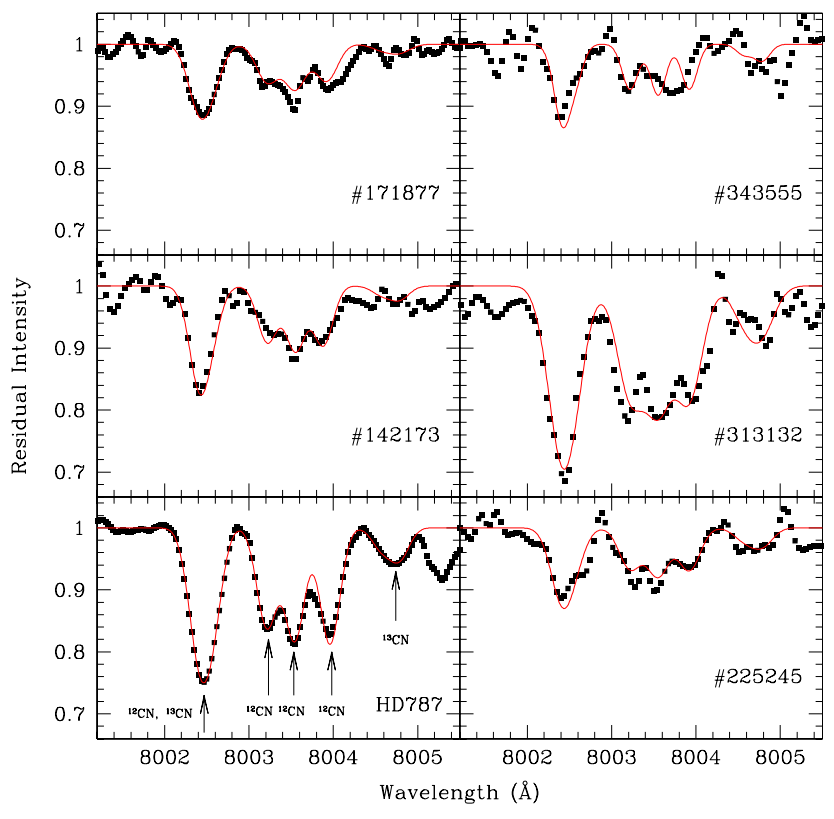

Fig. 3. Sample of the target stars' spectra in the region used to measure the $\mathrm{N}$ abundance and the ${ }^{12} \mathrm{C} /{ }^{13} \mathrm{C}$ isotopic ratio.

the relative intensity of the ${ }^{12} \mathrm{CN}$ or ${ }^{13} \mathrm{CN}$ molecular lines. For $\mathrm{Fe}$, the uncertainty owing to the noise is simply the error on the mean as given by MOOG. For the other elements, whose abundances were obtained by spectrosynthesis, the errors were evaluated by comparing observed spectra with synthetic ones calculated with a step of $0.05 \mathrm{dex}$ in abundance or 2 in ${ }^{12} \mathrm{C} /{ }^{13} \mathrm{C}$. We estimated by eye the abundance interval that is still compatible, within the noise, with the observed spectrum. The error owing to the noise was assumed to be the semi-amplitude of this interval.

As for the atmospheric parameters, uncertainties were estimated by changing one parameter at the time and measuring the abundances again. The error for a given parameter is given by the difference between the old and the new abundances. We assumed $\pm 100 \mathrm{~K}, \pm 0.2 \mathrm{dex}$, and $\pm 0.10 \mathrm{~km} \mathrm{~s}^{-1}$ as the typical errors for $T_{\text {eff }}, \log g$ and $\xi$, respectively, and analyzed stars \#313132 and \#225245, which cover the entire $T_{\text {eff }}$ range of our sample. Results are reported in Table 5. The last column in the table reports the total error, which is defined as

$$
\sigma_{\text {tot }}=\sqrt{\sigma_{S / N}^{2}+\sigma_{T_{\text {eff }}}^{2}+\sigma_{\log (g)}^{2}+\sigma_{\xi}^{2}} .
$$

\section{Rotational velocities}

The projected rotational velocity of the target stars, $v \sin i$, was measured from the width of the auto-cross correlation function (CCF) of their spectrum: each one was cross-correlated (Tonry \& Davis 1979) with itself through the fxcor IRAF task, and the peak of the resulting function was fitted with a Gaussian profile to derive its width $\sigma$. We verified that the resulting width changed when correlating different spectral ranges, but only by the addition of a color-dependent constant on all standard and target stars. This did not affect the measurements, since it only implied a different value for the calibrated constants of the $\sigma-v \sin i$ relation. We finally adopted the range 6000-6500 $\AA$, a high- $S / N$ region free of telluric bands and strong stellar lines, avoided because of their non-Gaussian wings.

The square of the width of the auto-CCF $\left(\sigma_{\text {obs }}\right)$ can be expressed as the quadratic sum of the rotational broadening $\left(\sigma_{\text {rot }}\right)$ and a term including all the causes of line broadening other than rotation $\left(\sigma_{0}\right)$. The relation between the measured $\sigma_{\mathrm{obs}}$ and the projected rotational velocity can thus be written as

$\sigma_{\text {rot }}^{2}=k(v \sin i)^{2}=\sigma_{\text {obs }}^{2}-\sigma_{0}^{2}$,

where $k$ is a constant coupling $v \sin i$ to the line rotational broadening. $\sigma_{0}$ is the sum of an instrumental constant and another term depending on the physical conditions in the stellar atmosphere (temperature, gravity, macro- and micro-turbulence). As a first approximation, $\sigma_{0}$ can be expressed as a function of color (Benz \& Mayor 1984; Queloz et al. 1998). Previous investigations showed that a linear relation between $\sigma_{0}$ and $(J-K)^{2}$ is the most appropriate (Melo et al. 2001) but, having at our disposal only five red giant standard observed for the calibration of the coefficients, we preferred the lower-order expression $\sigma_{0}^{2}=a+b(J-K)^{2}$. This approximation is allowed because 
Table 6. Projected rotational velocity derived for the standard stars.

\begin{tabular}{lcccc}
\hline \hline ID & $\begin{array}{c}v \sin i \text { (lit.) } \\
\mathrm{km} \mathrm{s}^{-1}\end{array}$ & $\begin{array}{c}v \sin i \text { (FEROS) } \\
\mathrm{km} \mathrm{s}^{-1}\end{array}$ & $v \sin i$ (duPont) & Ref. \\
\hline HD 787 $^{a}$ & $2.1 \pm 1.0$ & 1.3 & 1.5 & $1,2,3,4$ \\
& $2.1 \pm 1.0$ & - & 1.6 & \\
HD 31767 & $1.1 \pm 1.3$ & 2.0 & 2.3 & 2 \\
HD 180540 & $4.8 \pm 0.3$ & 4.7 & 4.8 & 9 \\
HD 4128 & $3.5 \pm 0.4$ & 3.6 & 3.4 & $5,6,7,8$ \\
HD 15453 & $1.0 \pm 1.0$ & 0.9 & 1.3 & 2 \\
\hline
\end{tabular}

Notes. The literature $v \sin i$, and the derived values for the FEROS and DuPont runs, are given in Cols. 2-4. ${ }^{(a)}$ For HD 787 only the FEROS spectrum obtained in the run reported in Table 1 was used for the abundance analysis. However, the star was repeatedly observed during the various duPont and La Silla runs. We report here the rotational velocities measured for the different spectra.

References. 1: de La Reza \& Da Silva (1995); 2: de Medeiros \& Mayor (1999); 3: de Medeiros et al. (1996); 4: Fekel \& Balachandran (1993); 5: Fekel (1997); 6: Gray (1982); 7: Pasquini et al. (2000); 8: Smith \& Dominy (1979); 9: Gray \& Toner (1986). When more than one estimate was available, the value in Col. 2 is their weighted average, with their standard deviation as associated error.

Table 7. Derived distances and orbital parameters of the program stars.

\begin{tabular}{|c|c|c|c|c|c|c|c|c|c|c|c|}
\hline SPM & $\begin{array}{c}R_{\odot} \\
\mathrm{kpc}\end{array}$ & $\begin{array}{c}X \\
\mathrm{kpc}\end{array}$ & $\begin{array}{c}Y \\
\mathrm{kpc}\end{array}$ & $\begin{array}{c}Z \\
\mathrm{kpc}\end{array}$ & $\begin{array}{c}U \\
\mathrm{~km} \mathrm{~s}^{-1}\end{array}$ & $\begin{array}{c}V \\
\mathrm{~km} \mathrm{~s}^{-1}\end{array}$ & $\begin{array}{c}W \\
\mathrm{~km} \mathrm{~s}^{-1}\end{array}$ & $e$ & $\begin{array}{c}Z_{\max } \\
\mathrm{kpc}\end{array}$ & $\begin{array}{c}R_{\mathrm{p}} \\
\mathrm{kpc}\end{array}$ & $\begin{array}{c}R_{\mathrm{a}} \\
\mathrm{kpc}\end{array}$ \\
\hline 142173 & $3.6 \pm 0.3$ & 7.92 & 0.47 & -3.48 & $-195 \pm 36$ & $41 \pm 39$ & $36 \pm 9$ & $0.83 \pm 0.02$ & $3.42 \pm 0.19$ & $1.13 \pm 0.01$ & $12.41 \pm 0.14$ \\
\hline 171877 & $3.1 \pm 0.3$ & 8.25 & 0.13 & -3.13 & $17 \pm 28$ & $-82 \pm 37$ & $27 \pm 3$ & $0.61 \pm 0.01$ & $2.19 \pm 0.06$ & $2.00 \pm 0.01$ & $8.27 \pm 0.06$ \\
\hline 225245 & $7.3 \pm 0.7$ & 8.55 & 0.10 & -7.28 & $56 \pm 41$ & $-52 \pm 43$ & $6 \pm 1$ & $0.76 \pm 0.03$ & $5.11 \pm 0.22$ & $1.60 \pm 0.02$ & $11.66 \pm 0.22$ \\
\hline 313132 & $0.7 \pm 0.1$ & 8.51 & 0.12 & -0.70 & $50 \pm 11$ & $-165 \pm 11$ & $12 \pm 2$ & $0.28 \pm 0.01$ & $0.67 \pm 0.01$ & $5.10 \pm 0.01$ & $9.09 \pm 0.01$ \\
\hline 343555 & $1.1 \pm 0.1$ & 8.60 & 0.14 & -1.07 & $33 \pm 9$ & $-29 \pm 20$ & $-84 \pm 3$ & $0.88 \pm 0.04$ & $1.93 \pm 0.10$ & $0.46 \pm 0.03$ & $7.37 \pm 0.15$ \\
\hline
\end{tabular}

our target stars are distributed in a narrow range of colors, and the systematics caused by a lower-order fit are expected to be negligible. In summary, renaming the involved constants appropriately, the relation between the measured $\sigma_{\mathrm{obs}}$ and $v \sin i$ can be written as

$(v \sin i)^{2}=A \sigma_{\mathrm{obs}}^{2}+B+C(J-K)^{2}$.

The coefficients $(A, B, C)$ were calibrated through a two-variables least-square procedure, by means of the five red giants standard observed during the same runs as our targets, with $(J-K)=$ $0.50-0.85$ and projected rotational velocity from the catalog of Glebocki \& Stawikowski (2000). The FEROS and duPont runs were calibrated separately, because of their different instrumental broadening.

The validity of the solution was tested comparing the $v \sin i$ of the standard stars calculated through Eq. (2) with its literature value. The results are given in Table 6 . The mean difference is negligible and the dispersion of the differences with literature values is $0.6 \mathrm{~km} \mathrm{~s}^{-1}$.

The rotational velocities measured for our program stars are given in Table 4 . The errors were estimated varying $(J-K)$, $\sigma_{\mathrm{obs}}$, and the input quantities of the calibration (color, $v \sin i$, and CCF width of the standard stars), by $\pm 1 \sigma$. The error on $(J-K)$ was taken from the 2MASS catalog, while an uncertainty of $0.05 \mathrm{~km} \mathrm{~s}^{-1}$ was assumed for the measured $\sigma$, according to Eq. (6) of Melo et al. (2001). For very slow rotators, $\sigma_{\text {obs }} \sim \sigma_{0}$ and measurement errors can cause $\sigma_{\text {obs }}<\sigma_{0}$, thus leading to an unphysical negative rotational velocity. This happened for two target stars. The upper limit of their $v \sin i$ was estimated as $v \sin i_{\max }=A \sqrt{2 \sigma_{0} \epsilon}$ (Melo et al. 2001), where $\epsilon$ is the uncertainty on $\sigma_{0}$, evaluated with the same procedure used to derive the errors on rotational velocity.

The derived projected rotational velocity is very low for all stars. This general result is confirmed by a simple inspection of the CCFs, because the $\sigma_{\text {obs }}$ of the targets never exceeded the analogous quantity for more than a couple of standard stars.
Although the width also depends on the spectral type, this indicates that $v \sin i$ of all the stars must be in the range spanned by the standards, i.e. $0-5 \mathrm{~km} \mathrm{~s}^{-1}$. The resulting errors are very similar to the dispersion of the differences with the literature value for the standard stars.

\section{Thick-disk membership}

The stars under analysis are drawn from a sample of likely thickdisk stars, as from the target selection criterion (see Sect. 2). Further insights into the actual nature of stars in our sub-sample can now be obtained by coupling the star kinematics to stellar isochrones and the estimated metallicities and atmospheric parameters.

We estimated the star distances as follows. For each star, we first generated an appropriate isochrone ${ }^{6}$ (Marigo et al. 2008) using the metallicities in Table 4. An age of $10 \mathrm{Gyr}$ was adopted for all stars at this stage (Feltzing \& Bensby 2009). Then, we inspected the corresponding isochrone searching for the best match of both gravity and effective temperature. This provides an estimate of the absolute magnitude which, together with the apparent magnitude and reddening in Table 2, allows to derive the star distances. Allowing for an uncertainty in the age of \pm 2 Gyr results in a typical error in distance of about $10 \%$. The derived distances are listed in Table 7 . For further reference, notice that a variation of $\Delta T_{\mathrm{eff}}= \pm 100 \mathrm{~K}$ and $\Delta \log g= \pm 0.5$ would result in a variation on the derived distances of about $4 \%$ and $17 \%$, in the case of \#142173 taken as representative of the stars under investigation.

To test the consistency of the possible thick-disk membership of the target stars, we explored their kinematical and orbital properties. Using the star distances in Table 7 and the star radial velocities (Table 3 ) and proper motions (Table 2), we estimated the Cartesian Galactic coordinates and velocity components

${ }^{6}$ http://stev.oapd.inaf.it/cgi-bin/cmd 


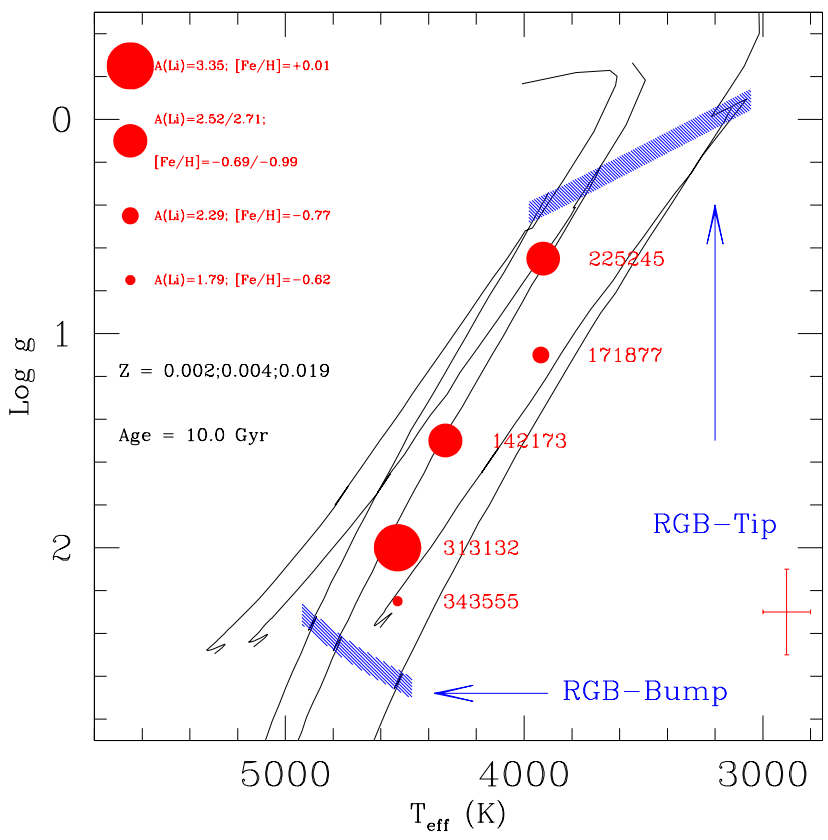

Fig. 4. Target stars are plotted in the $\log g$ vs. $T_{\text {eff }}$ plane together with $10 \mathrm{Gyr}$ theoretical isochrones of metallicity similar to the target stars. The symbol size is scaled according to the stars' Li content.

(see Table 7) following the method described by Johnson \& Soderblom (1987). Finally, we calculated the Galactic orbit of each star adopting a gravitational potential including the bar, deriving estimates for the eccentricity (e), the apo- and perigalacticon $\left(R_{\mathrm{a}}\right.$ and $\left.R_{\mathrm{p}}\right)$, and the absolute value of the maximum height above the plane $Z_{\max }$, which are also indicated in Table 7. Orbits were computed following the method described in Magrini et al. (2010) and Jilkova et al. (in prep.), which the reader is referred to for details.

Based on the above analysis, we can draw the following conclusions:

- according to the derived orbital parameters, all stars but \#313132 look like typical thick-disk stars;

- \#313132 has a low eccentricity and does not reach high $Z$. Besides, its orbit is confined within the solar vicinity. It is, therefore, reasonable to consider it as a thin-disk star. We note also that \#313132 presents a solar metallicity (see Table 4), which is typical of thin-disk stars.

\section{Discussion}

The abundances measured for the target stars are summarized in Table 4. All five stars have lithium abundances higher than $1.5 \mathrm{dex}$, and are, therefore, to be considered as Li-rich. Three of them present abundances exceeding 2.2 dex and one, namely $\# 313132$, belongs to the group of the rare super Li-rich giants (see, e.g., Balachandran et al. 2000; Domínguez et al. 2004; Reddy \& Lambert 2005; Monaco \& Bonifacio 2008; Kumar \& Reddy 2009).

In Fig. 4 we plot our stars in the $T_{\text {eff }}$ vs. $\log g$ plane, together with 10 Gyr theoretical isochrones (Marigo et al. 2008) spanning the metallicity range covered by our stars. Though they likely cover a range of ages, Feltzing \& Bensby (2009) concluded that thick-disk stars are older than the Sun with a mean age of 10 Gyr. The position of the RGB-bump and RGB-tip are marked in the figure for reference. For younger ages the RGB-bump position

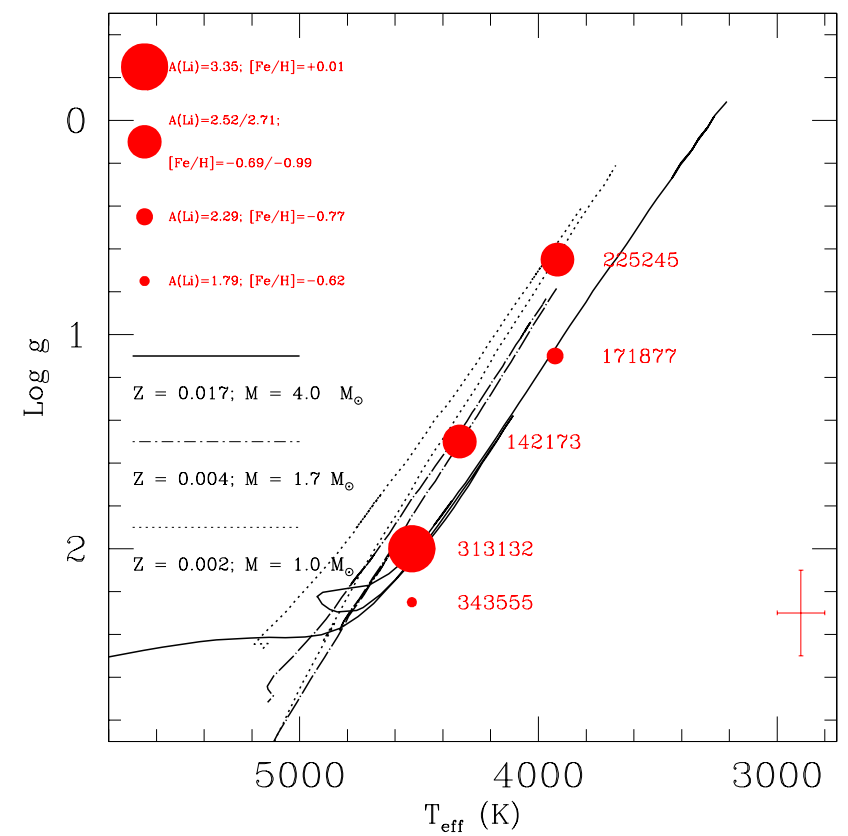

Fig. 5. Target stars are plotted in the $\log g$ vs. $T_{\text {eff }}$ plane together with theoretical evolutionary tracks of metallicity similar to the target stars and different masses. The symbol size is scaled according to the stars' Li content.

on the plane moves to cooler temperatures and lower gravities, but for ages as young as $3 \mathrm{Gyr}$, its location lies still within the shaded area in the figure.

As discussed in Sect. 5, \#313132 is likely to be a thin-disk star, and we will discuss its case separately. The other four stars seem to be more evolved than the RGB-bump, but lie below the RGB-tip. Their position in Fig. 4 would suggest they might have already suffered the extra-mixing process known to take place at the RGB-bump. While for stars \#142173,\#171877 and \#343555 we can only derive lower limits to the ${ }^{12} \mathrm{C} /{ }^{13} \mathrm{C}$ isotopic ratio, star \#225245 indeed presents a value lower than first dredge-up prediction for low-mass stars. If the Li-enrichment phase indeed occurs at the RGB-bump (CB00), this star may be in the process of diluting again their surface $\mathrm{Li}$ abundance and would be, therefore, similar to the HD $148293\left({ }^{12} \mathrm{C} /{ }^{13} \mathrm{C}=16, A(\mathrm{Li})=2\right)$ and HD 183492 cases $\left({ }^{12} \mathrm{C} /{ }^{13} \mathrm{C}=9, A(\mathrm{Li})=2\right)$ discussed by CB00. Following $\mathrm{CB} 00$, if it is indeed diluting its surface $\mathrm{Li}$, its position in Fig. 4 and its carbon isotopic ratios might provide constraints on the time-scale for the extra-mixing process to occur. At variance with HD 148293 and HD 183492, however, \#225245 is significantly more evolved than the RGB-bump and have high lithium abundances $(A(\mathrm{Li})>2.7)$.

Cool-bottom processing (CBP) could still be considered as a viable mechanism to produce the lithium abundances observed in these four stars. In particular, \#225245 has quite a high lithium abundance $(A(\mathrm{Li})>2.7)$. It lies close to the RGB-tip and has quite a low carbon isotopic ratio $\left({ }^{12} \mathrm{C} /{ }^{13} \mathrm{C}=8\right)$, similar to the predictions made by Boothroyd \& Sackmann (1999) for lowmass stars at the RGB-tip using a model of deep mixing and the associated CBP. These models were proved to be effective in producing Li-rich stars all along the RGB (Sackmann \& Boothroyd 1999 ) up to very high abundances, $A(\mathrm{Li})=4.0$. Considering its low metal content, \#225245 may also be similar to the Li-rich giants discovered in the globular clusters M 3 and NGC 362 (Kraft et al. 1999; Smith et al. 1999).

Together with our stars, we plot in Fig. 5 stellar evolutionary tracks (Bertelli et al. 2008, 2009) of metallicity appropriate 
to our stars. While the arguments in Sect. 5 cannot be considered as proof that they are thick-disk members, the locus occupied in the plane by the four stars we just discussed is consistent with them indeed being low-mass objects and, therefore, with the conclusions we have drawn above. We note that $\mathrm{CB} 00$ has shown that Li-rich giants cluster either at the RGB-bump or at the early AGB, in the case of low and intermediate-mass stars, respectively. Our stars, however, do not belong to either of these two categories.

It is clear from Fig. 5 that the atmospheric parameters of star \#313132 are perfectly compatible with a $4 M_{\odot}$ star. Its location in the plane is compatible with either being on the RGB or the AGB. This object may accordingly belong to the second group of Li-rich stars identified by CB00, i.e. stars on the early AGB, where the deepening of the envelope has not yet reached its maximum penetration and an extra-mixing process is supposed to occur to drive ${ }^{3} \mathrm{He}$ from the envelope to a region hot enough for the Cameron \& Fowler (1971) mechanism to take place. In this case, it would be similar to HD 787 and HD 30834, which also have similar ${ }^{12} \mathrm{C} /{ }^{13} \mathrm{C}$ isotopic ratios, compatible with first dredge-up predictions for similar masses (see discussion in CB00). Star \#313132 is, then, the first super Li-rich giant belonging to this group. Indeed, stars on the early AGB present on average lower $\mathrm{Li}$ abundances than stars at the RGB-bump. Guandalini et al. (2009) have interpreted this group of stars as actually lying on the RGB. Their models are unable to interpret super Li-rich stars though. We notice that it is in the 4-8 $M_{\odot}$ mass range that hot-bottom burning leading to $\mathrm{Li}$ production starts to became effective. This is known to occur at bolometric magnitudes $M_{\text {bol }} \simeq-6$ to -7 (Sackmann \& Boothroyd 1992; Smith et al. 1995). Theoretical tracks and isochrones (Bertelli et al. 2008, 2009; Marigo et al. 2008) suggest instead that atmospheric parameters similar to that of star \#313132 are compatible with significantly fainter luminosities only, namely $M_{\text {bol }} \simeq-2.1$ to -1.4 , for the following combination of parameters: $\left(T_{\mathrm{eff}}\right.$; $\log g$; mass $)=\left(4400-4600 \mathrm{~K} ; 1.8-2.2 ; 3.5-4.5 M_{\odot}\right)$. Therefore, the high lithium abundance of \#313132 should not have been reached through hot-bottom burning.

All our stars but \#343555 have Li abundances that exceed or are close to the maximum value allowed by the GPBU09 model for stars at the RGB-bump $(A(\mathrm{Li}) \simeq 2.5 \mathrm{dex})$, while more evolved stars would present even lower values.

As discussed in Sect. 1, the fraction of Li-rich giants seems to be highly enhanced among rapid rotators (Drake et al. 2002). The rotational velocities measured for our stars are reported in Table 4. All our targets are slow rotators, with only the super Li-rich star \#313132 presenting a slightly higher value, namely $3.3 \mathrm{~km} \mathrm{~s}^{-1}$. This value is, however, still well below the range of rotational velocities $\left(v \sin i>8 \mathrm{~km} \mathrm{~s}^{-1}\right)$ among which the frequency of Li-rich giants seems to increase.

In RGB stars, the Li-rich phase has also been suggested to be associated with a mass loss-episode (de La Reza et al. 1996, 1997). This latter can cause blue-shifted asymmetric $H_{\alpha}$ profiles or the presence of additional blue-shifted absorption lines in the Na doublet (Na D) lines (Balachandran et al. 2000; Drake et al. 2002; Reddy \& Lambert 2005) in the stellar spectra. Figures 6 and 7 present a sample of the spectra obtained for the target stars in the $\mathrm{H}_{\alpha}$ and $\mathrm{NaD}$ lines regions. Our stars do not present any evident asymmetry in the $\mathrm{H}_{\alpha}$ line (Fig. 6). \#142173 is the only star that presents additional blue shifted lines around the sodium doublet (Fig. 7). These additional components are blue-shifted by 40 and $39 \mathrm{~km} \mathrm{~s}^{-1}$ with respect to the companion photospheric $\mathrm{Na} \mathrm{D}$ lines. These velocities closely correspond to the local standard of rest $\left(v_{\text {helio }}=+38 \mathrm{~km} \mathrm{~s}^{-1}\right)$. Therefore, these features may

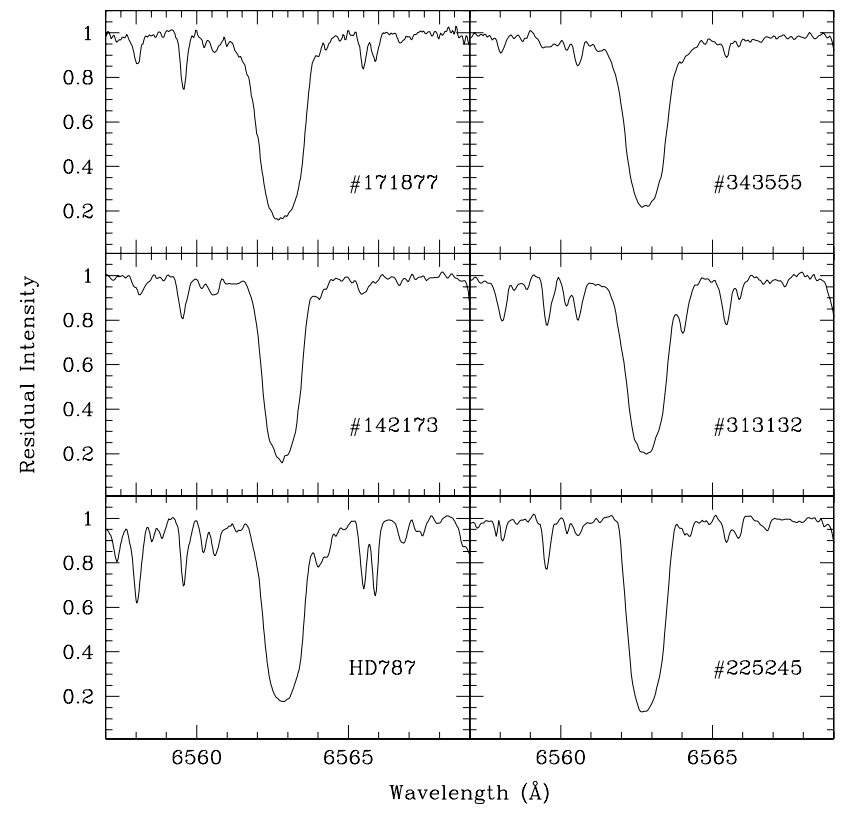

Fig. 6. Sample of the target stars' spectra in the region of the $\mathrm{H}_{\alpha}$ line.

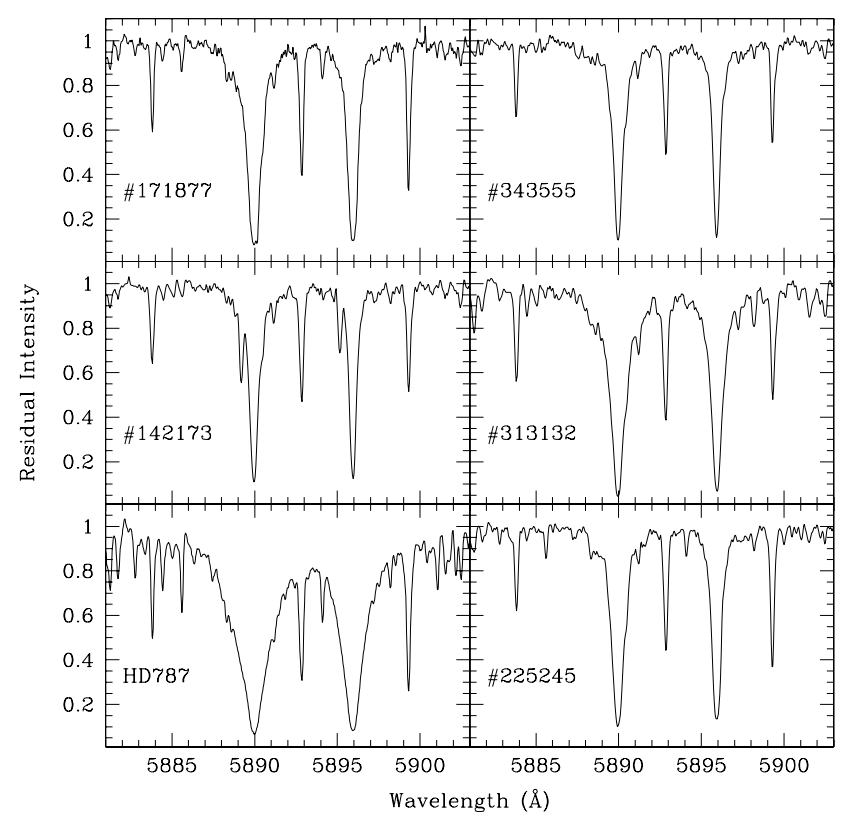

Fig. 7. Sample of the target stars' spectra in the region of the Na D lines.

originate in the interstellar medium, and not be of stellar origin (see, e.g., Balachandran et al. 2000).

\section{Conclusions}

We have built up a large database of high-resolution, highquality stellar spectra of candidate thick-disk giants. We searched it for stars presenting a strong Li I resonance line and detected five Li-rich giants. One of them, namely \#313132, has $A(\mathrm{Li})>3.3$ and therefore falls in the category of the rare super Li-rich giants (see Kraft et al. 1999; Domínguez et al. 2004; Monaco \& Bonifacio 2008; Kumar \& Reddy 2009; Carlberg et al. 2010).

All our stars but \#313132 have kinematics compatible with thick-disk membership, and a comparison with theoretical evolutionary tracks (see Fig. 5) of a proper metallicity is consistent 
with them being low-mass stars. They all seem to have evolved past the RGB-bump (Fig. 4). Therefore, they do not belong to either of the two evolutionary regions where Li-rich giants tend to cluster, namely the RGB-bump or the early AGB in low- and intermediate-mass stars, respectively. Figure 4 would suggest that these stars might have already undergone the extra-mixing process known to happen at the RGB-bump. The low carbon isotopic ratios measured in \#225245 may support this possibility, at least for this star. Under this hypothesis, they can be either produced by $\mathrm{CBP}$ or are in the process of diluting Li again after the enrichment phase. In the latter case, their ${ }^{12} \mathrm{C} /{ }^{13} \mathrm{C}$ isotopic ratios could be used as a diagnostic for the time scale involved in the dilution process after the Li-enrichment phase has occurred. \#225245 has a quite high lithium abundance $(A(\mathrm{Li})>2.7)$ and lies close to the RGB-tip. Its low ${ }^{12} \mathrm{C} /{ }^{13} \mathrm{C}$ isotopic ratio is consistent with the CBP predictions of Boothroyd \& Sackmann (1999).

\#313132 is a likely thin-disk member. Its spectroscopically derived atmospheric parameters are compatible with a $\sim 4 M_{\odot}$ star either in the RGB or AGB phase. It is the first super Li-rich giant detected at this phase (CB00, GPBU09). Its ${ }^{12} \mathrm{C} /{ }^{13} \mathrm{C}$ ratio is compatible with the first dredge-up predictions for similar masses.

The detected Li-rich stars are slow rotators and do not show indication of mass loss, as shown by the inspection of the $\mathrm{H}_{\alpha}$ and the $\mathrm{Na} \mathrm{D}$ lines.

Acknowledgements. C.M.B., S.V., and D.G. gratefully acknowledge support from the Chilean Centro de Astrofísica FONDAP No. 15010003 and the Chilean Centro de Excelencia en Astrofísica y Tecnologías Afines (CATA). M.Z. acknowledges Fondecyt Regular 1085278, the FONDAP Center for Astrophysics 15010003, Basal CATA PFB-06, and the MIDEPLAN Milky Way Millennium Nucleus P07-021-F. We thank Bruno Jungwiert for his input on a former version of the orbit integration code used in this contribution. Finally, we would like to thank the referee, Dr. P. North, for his helpful suggestions, which improved the quality of the present investigation.

\section{References}

Alonso, A., Arribas, S., \& Martínez-Roger, C. 1999, A\&AS, 140, 261 Balachandran, S. C., Fekel, F. C., Henry, G. W., \& Uitenbroek, H. 2000, ApJ, 542, 978

Benz, W., \& Mayor, M. 1984, A\&A, 138, 183

Bertelli, G., Girardi, L., Marigo, P., \& Nasi, E. 2008, A\&A, 484, 815

Bertelli, G., Nasi, E., Girardi, L., \& Marigo, P. 2009, A\&A, 508, 355

Boothroyd, A. I., \& Sackmann, I.-J. 1999, ApJ, 510, 232

Brown, J. A., Sneden, C., Lambert, D. L., \& Dutchover, E., Jr. 1989, ApJS, 71, 293

Cameron, A. G. W., \& Fowler, W. A. 1971, ApJ, 164, 111 (CF71)

Cardelli, J. A., Clayton, G. C., \& Mathis, J. S. 1989, ApJ, 345, 245

Carlberg, J. K., Smith, V. V., Cunha, K., Majewski, S. R., \& Rood, R. T. 2010, ApJ, 723, L103

Carraro, G., van Altena, W. F., Moni Bidin, C., et al. 2005, BAAS, 37, 1378

Castilho, B. V., Gregorio-Hetem, J., Spite, F., Barbuy, B., \& Spite, M. 2000, A\&A, 364, 674

Charbonnel, C., \& Balachandran, S. C. 2000, A\&A, 359, 563 (CB00)

Charbonnel, C., \& Do Nascimento, J. D., Jr. 1998, A\&A, 336, 915

Charbonnel, C., \& Zahn, J.-P. 2007, A\&A, 467, L15

da Silva, L., de La Reza, R., \& Barbuy, B. 1995, ApJ, 448, L41

de La Reza, R., \& da Silva, L. 1995, ApJ, 439, 917

de La Reza, R., Drake, N. A., \& da Silva, L. 1996, ApJ, 456, L115

de La Reza, R., Drake, N. A., da Silva, L., Torres, C. A. O., \& Martin, E. L. 1997, ApJ, 482, L77

de Medeiros, J. R., \& Mayor, M. 1999, A\&AS, 139, 433 de Medeiros, J. R., Melo, C. H. F., \& Mayor, M. 1996, A\&A, 309, 465

Denissenkov, P. A., \& Herwig, F. 2004, ApJ, 612, 1081

Denissenkov, P. A., \& Weiss, A. 2000, A\&A, 358, L49

Denissenkov, P. A., Pinsonneault, M., \& MacGregor, K. B. 2009, ApJ, 696, 1823

Domínguez, I., Abia, C., Straniero, O., Cristallo, S., \& Pavlenko, Y. V. 2004, A\&A, 422, 1045

Drake, N. A., de la Reza, R., da Silva, L., \& Lambert, D. L. 2002, AJ, 123, 2703

Fekel, F. C. 1997, PASP, 109, 514

Fekel, F. C., \& Balachandran, S. 1993, ApJ, 403, 708

Fekel, F. C., \& Watson, L. C. 1998, AJ, 116, 2466

Feltzing, S., \& Bensby, T. 2009, IAU Symp., 258, 23

Girard, T. M., Dinescu, D. I., van Altena, W. F., et al. 2004, AJ, 127, 3060

Girard, T. M., Korchagin, V. I., Casetti-Dinescu, D. I., et al. 2006, AJ, 132, 1768 (G06)

Glebocki, R., \& Stawikowski, A. 2000, Acta Astr., 50, 509

Gonzalez, O. A., et al. 2009, A\&A, 508, 289 (G09)

Gray D. F. 1982, ApJ, 262, 682

Gray D. F., \& Toner, C. G. 1986, ApJ, 310, 277

Gratton, R., Sneden, C., \& Carretta, E. 2004, ARA\&A, 42, 385

Grevesse, N., \& Sauval, A. J. 1998, Space Sci. Rev., 85, 161

Guandalini, R., Palmerini, S., Busso, M., \& Uttenthaler, S. 2009, PASA, 26, 168 (GPBU09)

Iben, I. J. 1967a, ApJ, 147, 624

Iben, I. J. 1967b, ApJ, 147, 650

Jasniewicz, G., Parthasarathy, M., de Laverny, P., \& Thévenin, F. 1999, A\&A, 342,831

Johansson, S., Litzén, U., Lundberg, H., \& Zhang, Z. 2003, ApJ, 584, L107

Johnson, D. R. H., \& Soderblom, D. R. 1987, AJ, 93, 864

Kraft, R. P., Peterson, R. C., Guhathakurta, P., et al. 1999, ApJ, 518, L53

Kumar, Y. B., \& Reddy, B. E. 2009, ApJ, 703, L46

Kupka, F. G., Ryabchikova, T. A., Piskunov, N. E., Stempels, H. C., \& Weiss, W. W. 2000, Baltic Astron., 9, 590

Kurucz, R. L. 1993, CD-ROM 13, 18, http: //kurucz.harvard.edu

Kurucz, R. L., Furenlid, I., Brault, J., \& Testerman, L. 1984, National Solar Observatory Atlas, Sunspot, New Mexico

Lèbre, A., Palacios, A., Do Nascimento, J. D., Jr., et al. 2009, A\&A, 504, 1011

Lind, K., Asplund, M., \& Barklem, P. S. 2009, A\&A, 503, 541

Magrini, L., Randich, S., Zoccali, M., et al. 2010, A\&A, 523, A11

Marigo, P., Girardi, L., Bressan, A., et al. 2008, A\&A, 482, 883

Marino, A. F., Villanova, S., Piotto, G., et al. 2008, A\&A, 490, 625 (M08)

Meléndez, J., Asplund, M., Alves-Brito, A., et al. 2008, A\&A, 484, L21

Melo, C. H. F., Pasquini, L., \& De Medeiros, J. R. 2001, A\&A, 375, 851

Melo, C. H. F., de Laverny, P., Santos, N. C., et al. 2005, A\&A, 439, 227

Monaco, L., \& Bonifacio, P. 2008, Mem. Soc. Astron. Ital., 79, 524

Moni Bidin, C. 2009, PhD Thesis

Moni Bidin, C., Carraro, G., Mendez, R. A., \& van Altena, W. F. 2010, ApJ, 724, L122

Pasquini, L., de Medeiros, J. R., \& Girardi, L. 2000, A\&A, 361, 1011

Queloz, D., Allain, S., Mermilliod, J.-C., Bouvier, J., \& Mayor, M. 1998, A\&A, 335,183

Reddy, B. E., \& Lambert, D. L. 2005, AJ, 129, 2831

Reddy, B. E., Lambert, D. L., Laws, C., Gonzalez, G., \& Covey, K. 2002, MNRAS, 335, 1005

Sackmann, I.-J., \& Boothroyd, A. I. 1992, ApJ, 392, L71

Sackmann, I.-J., \& Boothroyd, A. I. 1999, ApJ, 510, 217

Sbordone, L., Bonifacio, P., Castelli, F., \& Kurucz, R. L. 2004, Mem. Soc. Astron. Ital. Suppl., 5, 93

Schlegel, D. J., Finkbeiner, D. P., \& Davis, M. 1998, ApJ, 500, 525

Siess, L., \& Livio, M. 1999, MNRAS, 308, 1133

Skrutskie, M. F., et al. 2006, AJ, 131, 1163

Sneden, C. 1973, ApJ, 184, 839

Smith, M. A., \& Dominy, J. F. 1979, ApJ, 231, 477

Smith, V. V., Plez, B., Lambert, D. L., \& Lubowich, D. A. 1995, ApJ, 441, 735

Smith, V. V., Shetrone, M. D., \& Keane, M. J. 1999, ApJ, 516, L73

Sweigart, A. V., \& Mengel, J. G. 1979, ApJ, 229, 624

Tonry, J., \& Davis, M. 1979, AJ, 84, 1511

Uttenthaler, S., Lebzelter, T., Palmerini, S., et al. 2007, A\&A, 471, L41

Wyse, R. F. G., \& Gilmore, G. 2005, in Cozumel/Cancun conference on Resolved Stellar Populations, ASP Conf. Proc., ed. D. Valls-Gabaud, \& M. Chavez, eprint [arXiv: astro-ph/0510025] 